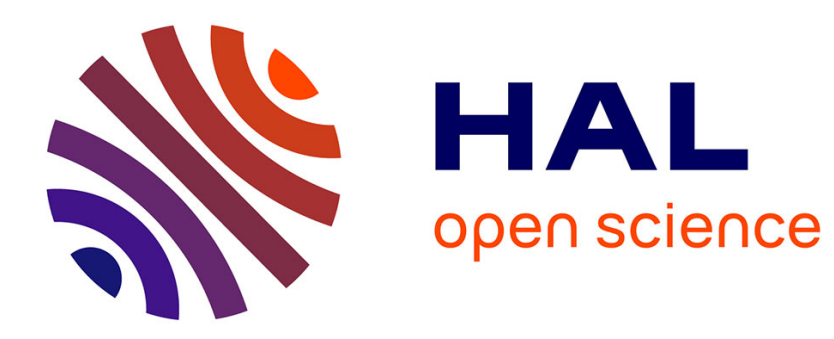

\title{
La structure et l'économie du Marais nantais
}

Jean-Marie Bourdeau

\section{To cite this version:}

Jean-Marie Bourdeau. La structure et l'économie du Marais nantais. Bulletin de l'Association de géographes français, 1936, 97, pp.75-82. 10.3406/bagf.1936.6986 . insu-01782536

\section{HAL Id: insu-01782536 \\ https://hal-insu.archives-ouvertes.fr/insu-01782536}

Submitted on 4 Mar 2021

HAL is a multi-disciplinary open access archive for the deposit and dissemination of scientific research documents, whether they are published or not. The documents may come from teaching and research institutions in France or abroad, or from public or private research centers.
L'archive ouverte pluridisciplinaire HAL, est destinée au dépôt et à la diffusion de documents scientifiques de niveau recherche, publiés ou non, émanant des établissements d'enseignement et de recherche français ou étrangers, des laboratoires publics ou privés.

\section{()ㅜ) $\Theta$}

Distributed under a Creative Commons Attribution - NoDerivatives| 4.0 International 


\section{La structure et l'économie du marais Nantais}

Jean-Marie Bourdeau

Citer ce document / Cite this document :

Bourdeau Jean-Marie. La structure et l'économie du marais Nantais. In: Bulletin de l'Association de géographes français, N97, 13e année, mai 1936. pp. 75-82;

doi : https://doi.org/10.3406/bagf.1936.6986

https://www.persee.fr/doc/bagf_0004-5322_1936_num_13_97_6986

Fichier pdf généré le 25/04/2018 
Communication de M. J.-M. Bourdeau

\section{La structure et l'économie du Marais nantais}

I. Le Paysage. - Au Nord de la Vendée, s'étend une région de terres amphibies, appelée très improprement Marais breton (1). Il occupe environ 45.000 hectares, à la limite des départements de Loire-Inférieure et de Vendée. Une sorte de presqu'île de micaschistes, qui s'avance presque jusqu'à la mer aux environs de Beauvoir, le coupe en deux parties qui reçoivent parfois des noms particuliers :

Au Nord, le Marais septentrional ou Marais de Bouin et de Machecoul, est situé au fond de la baie de Bourgneuf;

Le Marais occidental ou Marais de Mont, un peu plus étendu, se trouve au Sud de la presqu'ile schisteuse et se prolonge jusqu'aux environs de Saint-Hilaire-de-Riez et à l'embouchure de la Vie.

Cette région présente dans les pays de l'Ouest une physionomie particulière et elle possède, dans la vie vendéenne, une individualité marquée. A bien des rapports, en effet, elle diffère du Marais poitevin : tandis que celui-ci est entouré de plaines jurassiques qui lui sont presque étrangères, le Marais nantais se trouve, par sa structure même, en contact avec un reste de vieux massif : le Bocage vendéen. Antagonisme ou association, |les relations avec le Bocage commandent toute la vie du Marais et lui donnent sa physionomie propre.

C'est bien un contraste qui frappe tout d'abord : on a l'impression de deux mondes distincts. Quand, sur les chaussées récentes, coupées d'innombrables petits ponts en dos d'âne, on traverse le Marais, de Saint-Gilles à Bourgneuf, l'aspect change peu. C'est une étendue plate, rarement à plus de 4 mètres au-dessus du niveau de la mer. Les arbres sont rares dans le Marais de Mont, inexistants dans le Marais du Sud. Partout l'eau apparaît : dans les innombrables canaux, appelés «étiers》 (2), qui serpentent autour des champs, isolant les routes et les habitations; dans les prés toujours humides et dont le fond déprimé est souvent marécageux; dans l'atmosphère même, car, malgré une pluviosité moyenne (650 millimètres), l'humidité absolue est très grande, témoin la forte nébulosité et la brume fréquente qui augmente encore la mélancolie du paysage.

(1) On devrait parler de Marais vendéen, d'autant plus que le Marais du Sud, nommé Marais poitevin, ne pourrait être confondu avec lui, ou mieux de Marais nantais, étant donné l'attraction qu'il subit. L'appellation de Marais breton peut prêter à confusion.

(2) Le mot étier ou estier, qu'on trouve dans tous les vieux textes concernant lc pays, vient du latin aestarium. 
Si, au contraire, on suit une des routes qui vont de la cote vers l'intérieur, on découvre, presque sans transition, un paysage tout opposé. Ie contraste est parículièrement bien marqué sur la route de Saint-Jean-de-Mont a Challans : a quelques centaines de mètres d'intervalle, on a deux aspects différents. Après les étendues plates et sans arbres du Marais, on arrive tout à coup dans un pays bocager, avec les tètards de chènes ot d'ormeaux cntourant les champs, les cultures de blé et de choux, la vigne, les grandes étables perdues dans les arbres: on se croirait aux environs des Herbiers ou dans les Mauges.

Le contraste est brutal, el pourtant, derrière une haie, on apercoit, tout près, les étiers et les prés du Marais. On croit voir deux mondes étrangers l'un a l'autre: or leurs rapports sont nombreux. Bocage et Marais ont toujours été étroitement unis, et leurs liens apparaissent dans la structure, dans l'économie et dans l'habitat.

I1. La formation du sol. - Le Marais est de formation très recente et encore incomplite. Ies sédiments se sont déposés au fond d'un golfe limite par des collines schisteuses ef des dépots tertiaires qui montrent qu'il fut déjà comblé à l'auversien. On a d'ailleurs affaire à une région de stabilite médiocre qui a subi, sans doute, toutes les transgressions tertiaires.

Les alluvions ont été apportées par divers agents:

1" Ie principal est un courant de maré qui, venant du NorelOuest, a entraine dans la baie de Bourgneuf et au dela de l'ile de Noirmoutier les vases apportées par la loire. Ces vases n'ont pas eu encore le lemps de se deposer au fond de la mer, et, reprises par ce courant, elles viennent se fixcer sur la cote.

2" Les cours d'eau du bocage, peu importants mais trés nombreux, ont en hiver de's crues importantes: ansi les alluvions fluviales contribuent-elles au colmatage.

3" A la suite de Cavoleau (1), certains ont fait intervenir un courant de marée venu du Sud-()uest, qui, se chargeant de débris arraches par l'érosion marine - effectivement importante a la cote occidentale de Noirmoutier, les déposerait dans le golfe. S'il a une action, elle ne peut itre que tres reduite, car la forme dissymétricue du Marais montre des alluvions rares sur la rive soumise a ce courant (2).

4" On a aussi expliqué la formation du Marais de Mont, par un courant cotier du Sud, charge de vases de la Gironde. Hypo-

(1) Cavoldat : Statislique on description gindrale du dipartement de la Vendere. A Paris, chr\% Dumoulin, is 12 .

(2) Ie goulet de Fromentine, qui ferme la baic de bourgneuf atu Sud, est naturellenent le lieu de courants de maré trés nombreux et variés, d'oì lat facilite des hypothoses et leur danger: 
thèse fantaisiste, car le Marais - nous le savons par des documents sûrs - s'est formé derrière un cordon littoral qui le fermait au Sud et qui laissait au contraire les sédiments de la Loire pénétrer largement au Nord.

Ce colmatage se poursuit de nos jours el on peut prévoir l'évolution vers une còte rectiligne de la pointe Saint-Gildas aux Sables-d'Olonne. L'exploitation à commencé vers le Xi siècle, sous l'influence de grandes abbayes comme celles d'Orouet ou de Quinquenevent; installees sur d'anciennes îles, elles ont aménagé la région et accéléré les atterrissements. Au $x^{*}$ siècle, la vie économique était dejà intense et les « Ports de la Baie » (Bourgneuf, Prigny, etc.) faisaient avec la Hanse un commerce important. Au xvir" siecle, le pays avait l'aspect d'un vaste archipel très ramilic par des bras de mer assez larges pour entraver, en 1622, les opérations de Iouis XIII et Bassompierre contre les protestants de Soubise. Après un léger arrêt au xvin' siècle lié, comme en Flandre, à la situation politique - le colmatage s'est intensifié au $x r x^{n}$ siècle sous l'influence du préfet, de Barante, et surtout grâce aux efforts des « Socićtés de Marais », veritables wateringues, dont les présidents étaient élus et dirigeaient l'entretien cles etier's et l'aménagement des terres. Les dernières acquisitions ont eu lieu aux environs de Bouin où on a créé des polders : polders Saint-Céran en 1852 et polders du Jain en 1860. Actucllement, les grandes marées permettent se construire les levées qui fixent les vases et font reculer la mer, notamment aux environs de Beauvoir. Aussi voit-on les écluses récentes, naguère construites en bordure de la mer, se détacher a l'intérieur des terres. Toute cette évolution historique, visible dans la topongraphie, reflétéc dans les noms de lieux, donne au pays une originalité encore renforcée par la structure.

III. La structure. - - Soit au pied des collines schisteuses, soit au milien des alluvions, afleure le calcaire fendre de l'auversien, sous forme d'îlots dépassant rarement le niveau des alluvions (seule la butte de Sallertaine atteint 8 mòtres). Il constitue le fond du Marais où les sondages le trouvent à 5 ou 10 mètres de profondeur et plonge lentement sous la mer, car il apparait a marée basse au pont d'Yeu et dans les roches de bouin. Des lambeaux conserves sur le massif ancien montrent que la transgression cocène a dû ètre assez étenduc.

Ia présence de ces îlots permet plusieurs hypothèses: on peut songer à des effondrements gui auraient disloqué la surface tertiaire dont seuls certains fragments scraient restes au niveau primitif. Hypothèse peu vraisemblable si on considère que tout le Marais contient une trentaine de ces ilots, dont certains n'ont pas 100 mètres carrés, et qu'ils sont répartis sans 
direction maitresse apparente. D'autres explications, plus ou moins fantaisistes, ne rendent pas mieux compte des faits. Reste une lernière hypothèse faisant intervenir l'action d'un ancien réseau hydrographique. ['n retrait de la mer, diflicile à dater, aurait produit un abaissement du niveau de base et une reprise d'érosion sur la surface éocène. Ie calcaire très friable a clé creusé et la surface s'est abaissece par recoupement des petites vallées nombreuses en bordure du Bocage. Seules ont subsisté quelques buttes situes entre des cours d'eau moins importants ou constiluees de calcaire plus résistant (aujourd'hui encore ce calcairc est de dureté très variable). Lors de la transgression llandrienne, la mer a envahi le système de vallées et les alluvions se sont déposées dans les parties déblavées, ne laissant émorger que les buttes épargnécs par l'érosion. Cette bypothèse semble la plus satisfaisante : elle demande a ètre précisée et vérifié de façon rigoureuse, car il faut ètre particulièrement prudent dans une telle region où les modifications dues à la marée donnent parfois lillusion de transgressions el de régressions (1).

An Sud, le long du Marais de Mont, les dépots cenomaniens, grés et sables, s'appuient au Massif ancien et donnent un paysage pauvre très spécial. On y trouve de curieux dépots, qui n'ont jamais eté etudies de facon particulière: ce sont des silex décomposés et des cailloux a facettes, ainsi que des galets semblables a ceux de la rote. On peut dès lors songer à une pénéplaine posteretacé avec probabilité de climat aride, ou bien it une ancienne ligne de rivage, ce que suguere une forme topographique de falaise, très nette aux environs de Soullans.

Enfin tout l'ensemble est encadre par des blocs schisteux : ils apparaissent comme de veritables escarpements barrant l'horizon vers le Nord, malgré leur faible altitude (20 mètres au maximum) qui contraste avec les terres alluviales basses. On songe tout de suite à des escarpements de failles, car les altitudes diminuent sur les blocs dans la direction du Nord-Est et surtout parce qu'on trouve de part et d'autre des dépòts tertiaires identiques (auversien marin, auversien lacustre, etc...). Ainsi le bloc qui limite le Marais au Nord, se prolongeant juscu'a la pointe Saint-Gildas donne un escarpement très raide, atteignant parfois 35 mètres de dénivellation : il s'agirait d'un escarpement de faille, ce que confiment la carte geologicue (Nantes, 116), les minutes au 1/40.000" et la Carte Marine (2), oi l'on note, au pied de ce bloc, des profondeurs atteignant 10 mitres, alors que dans toute la baic de Bourgneuf, on ne trouve a

(1) D.-W. Jomssox : Ies valialions du nivealu de la mor et les modiflcations de la ligne de rivage tennedes de fieographie, 1.5 jamvier 1928).

(2) carte 5039 (G.). De la pointe de St-Gildas au goulet de liromentine. 
10 kilomètres de la côte que des fonds de .5 mètres. On peut donc songer à une série de blocs faillés et basculés vers le NordEst par des dislocations tertiaires postéricures à l'éocène qui passent vers le Sud à de simples flexures. Ces alignements se rattacheraient à ceux de la côte bretonne où on suit depuis penmarch et lile de Groix des lignes de dislocation avec compartiments basculćs vers l'intérieur.

IV. Les conditions d'exploitation. - Le Marais est donc individualisé par une structure très particulière - et dont on ne vient que d'énoncer quelques éléments. Son individualité est renforcée par un facteur économique : l'influence d'une région urbaine en plein essor, Nantes, dont, depuis peu d'années, l'action s'exerce dans cette direction et transforme l'économie du Marais pour on faire un de ses centres d'approvisionnement.

l'économic maraichine reste pourtant assujettie à la servitude séculaire que font peser sur elle des conditions d'exploitation très spéciales : le centre du Marais est recouvert par les caux en hiver et au début du printemps, le sol est parfois insuffisamment ameubli, ou bien la terre demande des attelages plus forts que ceux dont on dis?ose. L'élevage ne va pas sans difficulté, car l'hiver, il faut tenir le bétail à l'étable, et l'été, le sol desseché se fendille, lherbe est insuffisante et on doit recourir aux fourrages artificiels.

La période des hautes caux entrave la circulation : le sol est gorgé d'eau, les chemins deviennent vite impraticables: il ne reste qu'à les couner pour rendre les canaux utilisables. On voit combien la question des débouchés se pose alors de façon angoissante.

Pourtant le sol fertile (1) a tenté l'homme : à chaque difficulté il a trouvé une solution paliemment élaborée et perfectionnée, dont l'ensemble constitue une vie originale, faite d'ingéniosité et de ténacité.

Pour combattre l'excès d'humidité, les champs ont été aménagés en formes de dômes, les bossis; on les dispose en petiles planches ou en billons lègèrement bombés, séparés par les rigoles d'écoulement : ainsi voit-on au printemps l'eau claire briller entre les « mottées » ò les cultures sont déjà hautes.

Un mode de circulation original s'est développé : les moles, petits bateaux plats où le maraîchin se tient allongé et se glisse dans le dédale des canaux, les ningles, longues perches adroitement maniées, qui permettent de franchir d'un bond les fossés. Il n'était pas naguère jusclu'à l'habitation qui ne se fut ctroitement adaptée aux conditions naturelles: ce sont les

\footnotetext{
(1) C'est une argile marine à coquilles fossiles, dite bri ou argile à
} serobiculaires; elle est tres fertile quoiqu'un peu paure en chaux. 
bourrines, aux murs de terre battue et au toit de jonc, très basses et abritees du vent douest par un rideau de lamaris.

V. L'Economie Maraichine. - Surtout l'habilant ne s'est pas entèté à des cultures attardées, il s'est consacré à des spéculalions bien adaptecs, domnant des produits finis, faciles a transporter, et il a recours a des intermediaires pour parer au manque de débouches.

le ble est de plus en plus delaisse et on ne trouve guère comme cultures importantes que la luzerne ef la féverole, ared desi rendements très forts.

L'élevage est de loin la premiere ressource, avec 9j bètes a cornes pour 100 hectares. Il s'est développé grâce à une association entre Bocage of Marais: chaque proprietaire possède a la fois des «prés $\gg$ et des « champs». Sur le « pré » du Marais, pait le troupeau, dans le « champ 》 du Bocage on cultive le « vert » c'est-i-dire les fourrages qui permettent de nourrir le bétail pendant la sécheresse de l'été et les inondations de l'hiver. Ices animaux sont vendus à l'age de 3 ou 1 ans, dins le Bocage. Le beurre, fabricfue à la ferme, est expédic vers la region nantaise. Malheureusement la race bovine "maraichine » apparentéc aux Durham est mal fixée. Aussi lélevage du cheval attil longtemps domine: au xvm" siècle, les environs de St-fervais btaient un centre d'elevase de demi-sang lrès répute. Apres aroir atteint son apogee au milieu du xix" siecle, cet clevage perichita sous l'influence de la concurrence allemande, of surtoul du developpement de rautomobile. Pourtant or: assiste aujourdhui a un effort interessant : renoncant a derer le demi-san:s de vente diflicile, les elevemrs achètent des chevatix bretons de ${ }^{\prime}$ mois qu'ils revendent en poilou el en Sormandie après 2 ou 3 ans, speculalion avantageuse qui témoigne de lesprit d'enireprise des exploitants.

Mais un nouvel blevage a detrone totis les autres : celui des poulets ef cles canards. Le Mamis expedie 450.000 canards par an : cest le « canard nantais » eleve en liberte. Chaque paysan possede 20 a 30 mères canes gu'on fait couver en janvier et gui donnent 200 i 300 canclons vendus all printemps, represenlant un benelice de 10 a 15 frances piece. ("est une spéculation avantageuse qui $1{ }^{\circ}$ 'xige qu'un capilal minime, une petite exploitation et peu de soins. Aussi s'est-elle repandue dans tout le Marais oi elle a des consequenees curieuses : le peu d'importance de luniti de cullure entratue une dissemination en essaim de l'habital ef empeche l'abandon de la lerre, car les enfants penvent sinstalle: sans une srosse mise de fonds.

l'clevage des poulets est souvent complementaire du precédent el fournit un nouvel exemple de lassociation entre Bocage et Marais : a la limite des deux regions, le paysan possede à la 
fois des canards qui parcourent ses canaux et des poulets élevés en liberté sur le Bocage.

VI. Bocage et Marais. - Ainsi retrouvons-nous partout cette solidarite entre les deux pays; elle permet des associations avantageuses entre deux genres de vie: rien d'étonnant par conséquent à trouver bì une zone de recrudescence de l'habitat; la borlure du Bocage est frangede d'une ligne de fermes qui exploitent a la fois des champs et des prés. Les villages $y$ ent trouvé une almosphère un peu moins humide, des facilités de circulation et de debouches, des conditions favorables au commerce. Aus: i rencontre-ton tous les marches alignes en bordure du massif ancien : IBourgneuf-en-Retz et Beauvoir-surMer, anciens ports envasés, Machecoul, Saint-cervais et Challans, métropole du Marais, lype de la petite cite commercante de l'ouest, coquette et riche.

La prospésite du pays reside done dans le contact intime entre Marais et Bocage, resullat d'influences réciproques. La colonisation est partic du Bocage ; mais, au xrx" siècle, c'est le Marais qui a ranime le Bocage. Pourtant il ne s'est completement développé que lorsqu'il est bordé par un Bocage riche comme le Pays de lietz; ailleurs il est comme frappé d'inertie, quand apparaissent les sables cénomaniens infertiles. Il $y$ a là uil exemple frappant du: role que peuvent jouer l'une sur l'autre deux régions complementaires; on ne sait celle qui domine l'autre et leur association est le phénomène géographique essentiel. Sans le Bocage, le Marais trainerait une vie routinière; sans le Marais, le Bocage serait un « mauvais pays ».

VII. L'évolution moderne (1). - En tout cas, le Marais a une superiorité incontestable dans le regime de la propriété et de l'exploitation : la propriété y est très divisée et le nombre des pelits propriétaires a beaucoup augmenté : $600 / 0$ des exploitations ont une contenance moyenne de 1 ha. 75 ; par leur exiguite, elles ne sont avantageuses que si le propriétaire les exploite lui-même et, comme elles n'exigent pas un gros capital, beaucoup de petites gens deviennent propriétaires. Les autres sont fermiers, alors que le métayage domine dans le Bocage. Ainsi chanue ménage exploite lui-même sa propriété ou sa ferme et peut vivre sur 1 ou 2 hectares grâce à l' « industrie » du canard nantais qui exige peu de frais et peu d'outillage : un toit a canards, une «loge » en roseaux pour le cheval ou la rache, le tout construit par l'habitant. Parmi les pays de l'ouest, le Marais nantais est un de ceux où la terre est le plus

(1) Cous arons consulté à ce sujet une très pénétrante étude inédite de lecriain regionaliste Jean Yors idocteur Ronent, scinteur de la Vendé): "I a Population et l'Habitation rumales en Vendéc." 
exploitée : ni exode rural, ni dépopulation, ni dénatalité, telles en sont les conséquences bienfaisantes.

Enfin le Marais se modernise à sa façon : les genres de vie n'y ont gardé que les conditions archaicpues imposées a tout jamais par la nature. Une fois entre dans le reyon d'action de la région nantaise, il a amélioré scs formes dexploitation, organisé ses débouchés. Deux faits illustrent son évolution : d'abord l'électrification, ouvre diflicile dans un pays au sol meuble et à l'habitat extrèmement dispersé; d'autre part, la transformation de la maison : aux vieilles « bourrines » en terres battues, basses, sombres ct inconforlables, succèdent de coquettes maisons claires, au style de villas et aux noms ambiticux.

Le vieux paysage du Marais nantais où tout était sombre : la terre, les maisons, l'eau des canaux mal entretenus, jusqu'au bétail peu soigné, n'est plus qu'un souvenir. Il est remplacé par un visage qui respire la prospérité et une économic rationnelle : les taches blanches et rouges des fermes, l'eau claire des canaux où patrouillent les canards et la note archäique d'un moulin qui tourne au pied du Bocage.

D'une région sauvage of arriérée, l'evolution moderne, aidée par la ténacité et l'intelligence des habitants, a fait la partie la plus riche de la Vendée et une contrée d'avenir dans les pays de l'Ouest. 